\title{
Euler's Partition Theorem with Upper Bounds on Multiplicities
}

\author{
William Y. C. Chen \\ Center for Combinatorics, LPMC-TJKLC \\ Nankai University \\ Tianjin 300071, P.R. China \\ chen@nankai.edu.cn \\ Ae Ja Yee \\ Department of Mathematics \\ The Pennsylvania State University \\ University Park, PA 16802, USA \\ yee@math.psu.edu \\ Albert J. W. Zhu \\ China Ship Development and Design Center \\ Wuhan, Hubei 430064, P.R. China \\ jiawen.zhu2006@gmail.com
}

Submitted: May 6, 2012; Accepted: Sep 22, 2012; Published: Oct 4, 2012

Mathematics Subject Classifications: 05A17, 11P81

\begin{abstract}
We show that the number of partitions of $n$ with alternating sum $k$ such that the multiplicity of each part is bounded by $2 m+1$ equals the number of partitions of $n$ with $k$ odd parts such that the multiplicity of each even part is bounded by $m$. The first proof relies on two formulas with two parameters that are related to the four-parameter formulas of Boulet. We also give a combinatorial proof of this result by using Sylvester's bijection, which implies a stronger partition theorem. For $m=0$, our result reduces to Bessenrodt's refinement of Euler's partition theorem. If the alternating sum and the number of odd parts are not taken into account, we are led to a generalization of Euler's partition theorem, which can be deduced from a theorem of Andrews on equivalent upper bound sequences of multiplicities. Analogously, we show that the number of partitions of $n$ with alternating sum $k$ such that the multiplicity of each even part is bounded by $2 m+1$ equals the number of partitions of $n$ with $k$ odd parts such that the multiplicity of each even part is also bounded by $2 m+1$. We provide a combinatorial proof as well.
\end{abstract}

Keywords: partition, Euler's partition theorem, Sylvester's bijection 


\section{Introduction}

The main objective of this paper is to present a unification of a refinement and a generalization of Euler's partition theorem. More precisely, we show that the number of partitions of $n$ with alternating sum $k$ such that the multiplicity of each part is bounded by $2 m+1$ is equal to the number of partitions of $n$ with $k$ odd parts such that the multiplicity of each even part is bounded by $m$. The algebraic proof of this result relies on two formulas with two parameters that are related to the four-parameter formulas of Boulet on the generating function for partitions [9]. Meanwhile, we give a combinatorial proof by using Sylvester's bijection [16], which implies a stronger partition theorem.

For the case $m=0$, our result reduces to a refinement of Euler's partition theorem due to Bessenrodt [6]. From a different perspective, our result is also related to a theorem of Andrews [3] on equivalent upper bound sequences of multiplicities. If the alternating sum and the number of odd parts are not taken into account in our theorem, we obtain a connection between partitions with the multiplicity of each part bounded by $2 m+$ 1 and partitions with the multiplicity of each even parts bounded by $m$, which is a generalization of Euler's theorem. Indeed, it is a special case of a theorem of Andrews. In the language of Andrews, we may say that the upper bound sequence of multiplicities $(2 m+2,2 m+2,2 m+2, \ldots)$ is equivalent to the upper bound sequence of multiplicities $(\infty, m+1, \infty, m+1, \ldots)$. However, when the alternating sums and the number of odd parts are taken into consideration, there does not seem to be a general theorem on the equivalent upper bound sequences of multiplicities.

Based on the algebraic proof of the main result, we also show that the number of partitions of $n$ with alternating sum $k$ such that the multiplicity of each even part is bounded by $2 m+1$ equals the number of partitions of $n$ with $k$ odd parts such that the multiplicity of each even part is also bounded by $2 m+1$. We provide a combinatorial proof as well. In particular, for $m=0$, we find that the number of partitions of $n$ with alternating sum $k$ such that the even parts are distinct equals the number of partitions of $n$ with $k$ odd parts such that the even parts are distinct.

Let us give an overview of the theorem of Bessenrodt and the theorem of Andrews. Throughout this paper, we shall adopt the common notation on partitions used in Andrews [4]. A partition $\lambda$ of a positive integer $n$ is a finite sequence of positive integers $\lambda_{1}, \lambda_{2}, \ldots, \lambda_{r}$ such that $\lambda_{1} \geqslant \lambda_{2} \geqslant \cdots \geqslant \lambda_{r}$ and $\lambda_{1}+\lambda_{2}+\cdots+\lambda_{r}=n$. We write $\lambda=\left(\lambda_{1}, \lambda_{2}, \ldots, \lambda_{r}\right)$. Each $\lambda_{i}$ is called a part. The part $\lambda_{1}$ is called the largest part of $\lambda$. The number of parts of $\lambda$ is called the length of $\lambda$, denoted $l(\lambda)$. The weight of $\lambda$ is the sum of parts of $\lambda$, denoted $|\lambda|$. The conjugate partition of $\lambda$ is defined by $\lambda^{\prime}=\left(\lambda_{1}^{\prime}, \lambda_{2}^{\prime}, \ldots, \lambda_{t}^{\prime}\right)$, where $\lambda_{i}^{\prime}$ is the number of parts of $\lambda$ greater than or equal to $i$. The number of odd parts in $\lambda$ is denoted by $l_{o}(\lambda)$. The alternating sum of $\lambda$, namely,

$$
l_{a}(\lambda)=\lambda_{1}-\lambda_{2}+\lambda_{3}-\lambda_{4}+\cdots .
$$

is denoted by $l_{a}(\lambda)$.

Euler's partition theorem states that the number of partitions of $n$ into distinct parts is equal to the number of partitions of $n$ into odd parts. There are many refinements 
of Euler's partition theorem, see, for examples, Bessenrodt [6], Fine [11, pp.46-47] and Sylvester [4, p.24]. For $m=0$, our partition theorem reduces to a refinement of Euler's partition theorem due to Bessenrodt [6], which is a limiting case of the lecture hall theorem due to Bousquet-Mélou and Eriksson [7, 8]. Bessenrodt derived the following relation from Sylvester's bijection [16].

Theorem 1.1. The number of partitions of $n$ into distinct parts with alternating sum $l$ is equal to the number of partitions of $n$ into odd parts with length $l$. In terms of generating functions, we have

$$
\sum_{\lambda \in \mathcal{D}} y^{l_{a}(\lambda)} q^{|\lambda|}=\sum_{\mu \in \mathcal{O}} y^{l(\mu)} q^{|\mu|}
$$

where $\mathcal{D}$ denotes the set of partitions with distinct parts and $\mathcal{O}$ denotes the set of partitions with odd parts.

There is another refinement of Euler's partition theorem due to Glaisher [12]. A unification of the refinements of Bessenrodt [6] and Glaisher [12] has been obtained by Chen, Gao, Ji and Li [10] which can be deduced from Boulet's four-parameter formula [9]. A combinatorial proof of this unification is given in [10].

In another direction, there are several generalizations of Euler's partition theorem, see, for example, Alder [1], Andrews [2, 3, 5], Glaisher [12] and Moore [14]. Andrews [3] proved the following theorem on equivalent upper bound sequences of multiplicities, see also Pak [15]. An upper bound sequence of multiplicities is defined to be an infinite sequence $a=\left(a_{1}, a_{2}, a_{3}, \ldots\right)$ of nonnegative integers or infinity. Let $\operatorname{supp}(a)$ be the set of indices $i$ such that $a_{i}$ is finite. We say that two upper bound sequences of multiplicities $a=\left(a_{1}, a_{2}, a_{3}, \ldots\right)$ and $b=\left(b_{1}, b_{2}, b_{3}, \ldots\right)$ are equivalent, if there exists a one-to-one correspondence $\pi: \operatorname{supp}(a) \rightarrow \operatorname{supp}(b)$ such that $i a_{i}=j b_{j}$, for all $j=\pi(i)$.

Theorem 1.2. Let $a=\left(a_{1}, a_{2}, a_{3}, \ldots\right)$ and $b=\left(b_{1}, b_{2}, b_{3}, \ldots\right)$ be two upper bound sequences of multiplicities. If $a$ and $b$ are equivalent, then the number of partitions $\lambda=$ $\left(1^{m_{1}} 2^{m_{2}} \cdots\right)$ of $n$ such that $m_{i}<a_{i}$ for all $i$ equals the number of partitions $\mu=$ $\left(1^{m_{1}} 2^{m_{2}} \cdots\right)$ of $n$ such that $m_{i}<b_{i}$ for all $i$.

In the language of Andrews, we may say that the upper bound sequence of multiplicities $a=(2 m+2,2 m+2,2 m+2, \ldots)$ is equivalent to the upper bound sequence of multiplicities $b=(\infty, m+1, \infty, m+1, \ldots)$. To be more specific, let $\pi$ be the map $i \rightarrow 2 i$, then we have $i a_{i}=\pi(i) b_{\pi(i)}$. In this case, we have the following consequence, which can be considered as a generalization of Euler's partition theorem.

Corollary 1.3. The number of partitions of $n$ with the multiplicity of each part bounded by $2 m+1$ equals the number of partitions of $n$ with the multiplicity of each even part bounded by $m$.

It can be seen that our main result is a unification of Theorem 1.1 and Corollary 1.3. This paper is organized as follows. In Section 2, we provide two formulas on twoparameter generating functions for partitions that are related to Boulet's four-parameter 
formula by imposing an upper bound sequence of multiplicities. In Section 3, we use the two formulas presented in Section 2 to prove the main theorem. We also prove the second result of this paper, that is, the number of partitions of $n$ with alternating sum $k$ such that each even part appears at most $2 m+1$ times equals the number of partitions of $n$ with $k$ odd parts such that each even part appears at most $2 m+1$ times. In Section 4, we give a combinatorial proof of the main result based on Sylvester's bijection and obtain a stronger version concerning the number of odd parts, the largest odd part and the largest part with odd multiplicity. Finally, we provide a combinatorial proof of our second theorem in the last section.

\section{Two-parameter Formulas}

To prove the main result of this paper, we need two formulas on two-parameter generating functions for partitions. The first formula (Theorem 2.3) can be deduced from Boulet's four-parameter formula [9]. It seems that there are typos in the formula of Boulet. A corrected version with a technical condition added is presented in Theorem 2.2. The first two-parameter formula (Theorem 2.3) can be deduced from Theorem 2.2 for the case $i=0, k=1, a=b$ and $c=d$. We provide the second two-parameter formula (Theorem 2.4) from a two-parameter formula which is a specialization of Theorem 2.1 by setting $a=c$ and $b=d$. The proof is similar to that of Boulet's four-parameter formula [9]. It should be noted that Theorem 2.3 can be considered as an extension of the two-parameter formula obtained from Theorem 2.1 by setting $a=b$ and $c=d$. The proof of the first two-parameter formula (Theorem 2.3) is analogous to that of Theorem 2.4, and hence it is omitted.

To state Boulet's four-parameter formula, let $\lambda$ be a partition, and let $a, b, c, d$ be commuting indeterminants. Define

$$
\omega(\lambda)=a^{\sum_{i \geqslant 1}\left\lceil\lambda_{2 i-1} / 2\right\rceil} b^{\sum_{i \geqslant 1}\left\lfloor\lambda_{2 i-1} / 2\right\rfloor} c^{\sum_{i \geqslant 1}\left\lceil\lambda_{2 i} / 2\right\rceil} d^{\sum_{i \geqslant 1}\left\lfloor\lambda_{2 i} / 2\right\rfloor} .
$$

The above four-parameter weight is introduced by Boulet [9]. Let

$$
\Phi(a, b, c, d)=\sum_{\lambda} \omega(\lambda)
$$

where the sum is over all partitions. Boulet [9] obtained the following formula.

Theorem 2.1. We have

$$
\Phi(a, b, c, d)=\prod_{j=1}^{\infty} \frac{\left(1+a^{j} b^{j-1} c^{j-1} d^{j-1}\right)\left(1+a^{j} b^{j} c^{j} d^{j-1}\right)}{\left(1-a^{j} b^{j} c^{j} d^{j}\right)\left(1-a^{j} b^{j} c^{j-1} d^{j-1}\right)\left(1-a^{j} b^{j-1} c^{j} d^{j-1}\right)} .
$$

Boulet generalized the above formula by considering an upper bound sequence of multiplicities. We shall adopt the notation in Boulet [9]. Let $R$ be a subset of positive integers, and let $\rho$ be a map from $R$ to the set $E$ of even positive integers. Boulet defined 
$\operatorname{Par}(i, k ; R, \rho)$ as the set of partitions $\lambda$ with parts congruent to $i(\bmod k)$ such that for any $r \in R$ the multiplicity of $r$ in $\lambda$ is less than $\rho(r)$. In other words, $\rho$ can be viewed as an upper bound sequence of multiplicities for partitions in $\operatorname{Par}(i, k ; R, \rho)$. Let

$$
\Phi_{i, k ; R, \rho}(a, b, c, d)=\sum_{\lambda} \omega(\lambda)
$$

where the sum is over all partitions in $\operatorname{Par}(i, k ; R, \rho)$.

Boulet [9] derived a formula for $\Phi_{i, k ; R, \rho}(a, b, c, d)$, which seems to contain typos. This formula can be corrected as follows. First, we impose a further condition on the definition of $\operatorname{Par}(i, k ; R, \rho)$, that is, for $i \neq 0$, the length of $\lambda$ is even and the part $i$ appears at most once. Then the formula for $\Phi_{i, k ; R, \rho}(a, b, c, d)$ takes the following form.

Theorem 2.2. We have

$$
\Phi_{i, k ; R, \rho}(a, b, c, d)=S T
$$

where

$$
S=\prod_{j=1}^{\infty} \frac{\left(1+a^{\left\lceil\frac{j k+i}{2}\right\rceil} b^{\left\lfloor\frac{j k+i}{2}\right\rfloor} c^{\left\lceil\frac{(j-1) k+i}{2}\right\rceil} d^{\left\lfloor\frac{(j-1) k+i}{2}\right\rfloor}\right)}{\left(1-a^{\left\lceil\frac{j k+i}{2}\right\rceil} b^{\left\lfloor\frac{j k+i}{2}\right\rfloor} c^{\left\lceil\frac{j k+i}{2}\right\rceil} d^{\left\lfloor\frac{j k+i}{2}\right\rfloor}\right)\left(1-a^{j k} b^{j k} c^{(j-1) k} d^{(j-1) k}\right)}
$$

and

$$
T=\prod_{r \in R}\left(1-a^{\left\lceil\frac{r}{2}\right\rceil \frac{\rho(r)}{2}} b^{\left\lfloor\frac{r}{2}\right\rfloor \frac{\rho(r)}{2}} c^{\left\lceil\frac{r}{2}\right\rceil \frac{\rho(r)}{2}} d^{\left\lfloor\frac{r}{2}\right\rfloor \frac{\rho(r)}{2}}\right) .
$$

For the purpose of this paper, we only need the special case of $(2.5)$, which is the case for $k=1, i=0, a=b$ and $c=d$. For this special case, we define

$$
\omega_{1}(\lambda)=a^{\sum_{i \geqslant 1}\left\lceil\lambda_{2 i-1} / 2\right\rceil} a^{\sum_{i \geqslant 1}\left\lfloor\lambda_{2 i-1} / 2\right\rfloor} b^{\sum_{i \geqslant 1}\left\lceil\lambda_{2 i} / 2\right\rceil} b^{\sum_{i \geqslant 1}\left\lfloor\lambda_{2 i} / 2\right\rfloor} .
$$

For notational simplicity, we denote by $\operatorname{Par}(R, \rho)$ the set of partitions such that any element $r$ in $R$ appears as a part with multiplicity less than $\rho(r)$. Set

$$
\Phi_{R, \rho}(a, a, b, b)=\Phi_{0,1 ; R, \rho}(a, a, b, b)=\sum_{\lambda} \omega_{1}(\lambda)
$$

where the sum is over all partitions in $\operatorname{Par}(R, \rho)$. We can deduce the following formula from $(2.5)$.

Theorem 2.3. We have

$$
\begin{aligned}
\Phi_{R, \rho}(a, a, b, b)= & \prod_{j=1}^{\infty} \frac{\left(1+a^{\left\lceil\frac{j}{2}\right\rceil+\left\lfloor\frac{j}{2}\right\rfloor} b^{\left\lceil\frac{j-1}{2}\right\rceil+\left\lfloor\frac{j-1}{2}\right\rfloor}\right)}{\left(1-a^{\left\lceil\frac{j}{2}\right\rceil+\left\lfloor\frac{j}{2}\right\rfloor} b^{\left\lceil\frac{j}{2}\right\rceil+\left\lfloor\frac{j}{2}\right\rfloor}\right)\left(1-a^{2 j} b^{2 j-2}\right)} \\
& \times \prod_{r \in R}\left(1-a^{\left(\left\lceil\frac{r}{2}\right\rceil+\left\lfloor\frac{r}{2}\right\rfloor\right) \frac{\rho(r)}{2}} b^{\left(\left\lceil\frac{r}{2}\right\rceil+\left\lfloor\frac{r}{2}\right\rfloor\right) \frac{\rho(r)}{2}}\right) .
\end{aligned}
$$


Bear in mind that the condition that $\rho(r)$ is even for any $r \in R$ is required in Theorem 2.3. However, as will be seen, we can obtain similar formulas for any upper bound sequence of multiplicities as long as we have $a=c$ and $b=d$. So we define

$$
\omega_{2}(\lambda)=a^{\sum_{i \geqslant 1}\left\lceil\lambda_{2 i-1} / 2\right\rceil} b^{\sum_{i \geqslant 1}\left\lfloor\lambda_{2 i-1} / 2\right\rfloor} a^{\sum_{i \geqslant 1}\left\lceil\lambda_{2 i} / 2\right\rceil} b^{\sum_{i \geqslant 1}\left\lfloor\lambda_{2 i} / 2\right\rfloor} .
$$

To be more specific, let $\gamma$ be a map from $R$ to positive integers. Let

$$
\Psi_{R, \gamma}(a, b, a, b)=\sum_{\lambda} \omega_{2}(\lambda)
$$

where the sum is over partitions in $\operatorname{Par}(R, \gamma+1)$. To derive our main theorem, we need the following extension of a two-parameter formula, that is, the generating function for $\Psi_{R, \gamma}(a, b, a, b)$. The proof of the following two-parameter formula is analogous to that of Boulet's four-parameter formula [9].

Theorem 2.4. We have

$$
\Psi_{R, \gamma}(a, b, a, b)=U V
$$

where

$$
U=\prod_{j=1}^{\infty} \frac{\left(1+a^{\left\lceil\frac{j}{2}\right\rceil} b^{\left\lfloor\frac{j}{2}\right\rfloor} a^{\left\lceil\frac{j-1}{2}\right\rceil} b^{\left\lfloor\frac{j-1}{2}\right\rfloor}\right)}{\left(1-a^{2\left[\frac{j}{2}\right\rceil} b^{2\left\lfloor\frac{j}{2}\right\rfloor}\right)\left(1-a^{2 j-1} b^{2 j-1}\right)}
$$

and

$$
V=\prod_{r \in R}\left(1-a^{\left\lceil\frac{r}{2}\right\rceil(\gamma(r)+1)} b^{\left\lfloor\frac{r}{2}\right\rfloor(\gamma(r)+1)}\right) .
$$

Proof. In view of the definition of $\Psi_{R, \gamma}(a, b, c, d)$, it is easy to see that $\Psi_{\emptyset, \gamma}(a, b, a, b)$ equals $\Phi(a, b, a, b)$ as given in Theorem 2.1. Hence we obtain

$$
\begin{aligned}
\Psi_{\emptyset, \gamma}(a, b, a, b) & =\prod_{j=1}^{\infty} \frac{\left(1+a^{j} b^{j-1} a^{j-1} b^{j-1}\right)\left(1+a^{j} b^{j} a^{j} b^{j-1}\right)}{\left(1-a^{j} b^{j} a^{j} b^{j}\right)\left(1-a^{j} b^{j} a^{j-1} b^{j-1}\right)\left(1-a^{j} b^{j-1} a^{j} b^{j-1}\right)} \\
& =\prod_{j=1}^{\infty} \frac{\left(1+a^{\left\lceil\frac{j}{2}\right\rceil} b^{\left\lfloor\frac{j}{2}\right\rfloor} a^{\left\lceil\frac{j-1}{2}\right\rceil} b^{\left\lfloor\frac{j-1}{2}\right\rfloor}\right)}{\left(1-a^{2\left\lceil\frac{j}{2}\right\rceil} b^{2\left\lfloor\frac{j}{2}\right\rfloor}\right)\left(1-a^{2 j-1} b^{2 j-1}\right)},
\end{aligned}
$$

which is the generating function for $\operatorname{Par}(\emptyset, \gamma+1)$.

In order to obtain the generating function for $\operatorname{Par}(R, \gamma+1)$, we notice that any partition $\lambda$ in $\operatorname{Par}(\emptyset, \gamma+1)$ can be expressed as a bipartition $(\mu, \nu)$ such that $\mu \in \operatorname{Par}(R, \gamma+1)$ and $\nu \in \mathcal{L}$, where $\mathcal{L}$ is the set of partitions with parts $r \in R$ occurring a multiple of $\gamma(r)+1$ times. In other words, there is a bijection between $\operatorname{Par}(\emptyset, \gamma+1)$ and $\operatorname{Par}(R, \gamma+1) \times \mathcal{L}$. Clearly, any part $r \in R$ occurring a multiple of $\gamma(r)+1$ times can be represented as a multiple of the following block

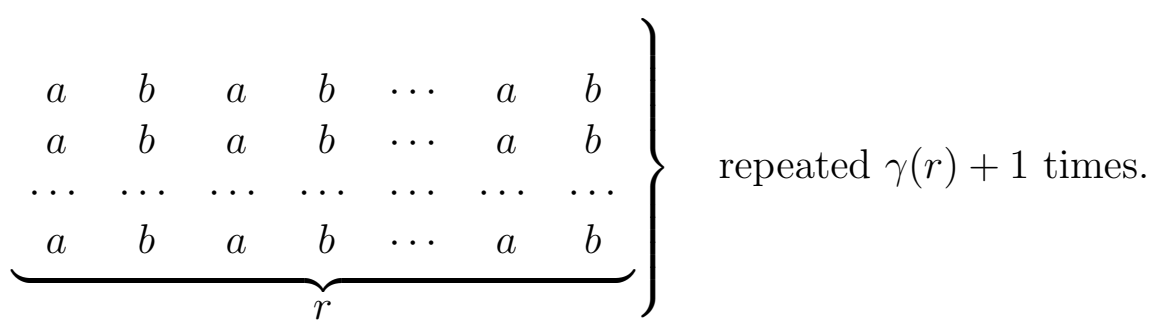


The weight of the above block is $a^{\left\lceil\frac{r}{2}\right\rceil(\gamma(r)+1)} b^{\left\lfloor\frac{r}{2}\right\rfloor(\gamma(r)+1)}$. Thus the generating function for $\mathcal{L}$ equals

$$
V^{-1}=\prod_{r \in R} \frac{1}{\left(1-a^{\left\lceil\frac{r}{2}\right\rceil(\gamma(r)+1)} b^{\left\lfloor\frac{r}{2}\right\rfloor(\gamma(r)+1)}\right)}
$$

It follows that

$$
\Psi_{R, \gamma}(a, b, a, b)=U V
$$

This completes the proof.

\section{Two Partition Theorems}

In this section, we provide two partition theorems. The first one is stated as follows.

Theorem 3.1. For $k, m, n \geqslant 0$, the number of partitions of $n$ with alternating sum $k$ such that each part appears at most $2 m+1$ times equals the number of partitions of $n$ with $k$ odd parts such that each even part appears at most $m$ times.

For example, the following table illustrates the case for $n=7$ and $m=1$.

\begin{tabular}{|c|c||c|c|}
\hline$l_{a}$ & Each part appears at most three times & $l_{o}$ & Each even part appears only once \\
\hline 1 & $\left(2^{2}, 1^{3}\right)\left(2^{3}, 1\right)\left(3,2,1^{2}\right)\left(3^{2}, 1\right)(4,3)$ & 1 & $(4,2,1)(4,3)(5,2)(6,1)(7)$ \\
\hline 3 & $\left(3,2^{2}\right)\left(4,1^{3}\right)(4,2,1)(5,2)$ & 3 & $\left(3,2,1^{2}\right)\left(3^{2}, 1\right)\left(4,1^{3}\right)\left(5,1^{2}\right)$ \\
\hline 5 & $\left(5,1^{2}\right)(6,1)$ & 5 & $\left(2,1^{5}\right)\left(3,1^{4}\right)$ \\
\hline 7 & $(7)$ & 7 & $\left(1^{7}\right)$ \\
\hline
\end{tabular}

If the alternating sum and the number of odd parts are not taken into account, we are led to Corollary 1.3, which can be regarded as a generalization of Euler's partition theorem and a special case of Theorem 3.1. Meanwhile, it is easy to see that Theorem 3.1 reduces to Theorem 1.1 when $m=0$.

The second partition theorem is concerned with the alternating sum, the number of odd parts and an upper bound $2 m+1$ on the multiplicity of each even part.

Theorem 3.2. For $k, m, n \geqslant 0$, the number of partitions of $n$ with alternating sum $k$ such that each even part appears at most $2 m+1$ times equals the number of partitions of $n$ with $k$ odd parts such that each even part appears at most $2 m+1$ times.

For example, for $n=7$ and $m=0$, the partitions in Theorem 3.2 are listed in the following table.

\begin{tabular}{|c|c||c|c|}
\hline$l_{a}$ & Each even part appears only once & $l_{o}$ & Each even part appears only once \\
\hline 1 & $\left(1^{7}\right)\left(2,1^{5}\right)\left(3,2,1^{2}\right)\left(3^{2}, 1\right)(4,3)$ & 1 & $(4,2,1)(4,3)(5,2)(6,1)(7)$ \\
\hline 3 & $\left(3,1^{4}\right)\left(4,1^{3}\right)(4,2,1)(5,2)$ & 3 & $\left(3,2,1^{2}\right)\left(3^{2}, 1\right)\left(4,1^{3}\right)\left(5,1^{2}\right)$ \\
\hline 5 & $\left(5,1^{2}\right)(6,1)$ & 5 & $\left(2,1^{5}\right)\left(3,1^{4}\right)$ \\
\hline 7 & $(7)$ & 7 & $\left(1^{7}\right)$ \\
\hline
\end{tabular}


To give the proofs of the above theorems, we shall adopt the common notation for the $q$-shifted factorials

$$
(a ; q)_{n}=(1-a)(1-a q) \cdots\left(1-a q^{n-1}\right), \quad n \geqslant 1,
$$

and

$$
(a ; q)_{\infty}=\lim _{n \rightarrow \infty}(1-a)(1-a q)\left(1-a q^{2}\right) \cdots,
$$

where $0 \leqslant|q|<1$, see Gasper and Rahman [13].

Notice that the generating functions for partitions in Theorem 3.1 and Theorem 3.2 can be expressed in the notation $\Phi_{R, \rho}(a, b, c, d)$ and $\Psi_{R, \gamma}(a, b, c, d)$ defined in the previous section, where $\rho$ and $\gamma$ are upper bound sequences of multiplicities. More precisely, $\Phi_{R, \rho}(a, b, c, d)$ is the generating function for partitions such that each part $r \in R$ appears less than $\rho(r)$ times with $\rho(r)$ being an even number, and $\Psi_{R, \gamma}(a, b, c, d)$ is the generating function for partitions such that each part $r \in R$ appears at most $\gamma(r)$ times.

In the above notation, it is easy to check that

$$
\Phi_{\mathbb{N}, 2 m+2}\left(x q, x q, x^{-1} q, x^{-1} q\right)
$$

is the generating function for partitions such that each part appears at most $2 m+1$ times and the exponent of $x$ is the alternating sum. We also observe that

$$
\Psi_{E, m}\left(x q, x^{-1} q, x q, x^{-1} q\right)
$$

is the generating function for partitions such that each even part appears at most $m$ times and the exponent of $x$ is the number of odd parts.

Based on the above interpretations of the functions $\Phi_{\mathbb{N}, 2 m+2}\left(x q, x q, x^{-1} q, x^{-1} q\right)$ and $\Psi_{E, m}\left(x q, x^{-1} q, x q, x^{-1} q\right)$, one can deduce Theorem 3.1 from the following relation.

Theorem 3.3. We have

$$
\Phi_{\mathbb{N}, 2 m+2}\left(x q, x q, x^{-1} q, x^{-1} q\right)=\Psi_{E, m}\left(x q, x^{-1} q, x q, x^{-1} q\right)=\frac{\left(-x q ; q^{2}\right)_{\infty}\left(q^{2 m+2} ; q^{2 m+2}\right)_{\infty}}{\left(q^{2} ; q^{2}\right)_{\infty}\left(x^{2} q^{2} ; q^{4}\right)_{\infty}} .
$$

Proof. Setting $a=x q, b=x q, R=\mathbb{N}$, and $\rho=2 m+2$ in Theorem 2.3, we obtain

$$
\begin{aligned}
& \Phi_{\mathbb{N}, 2 m+2}\left(x q, x q, x^{-1} q, x^{-1} q\right) \\
& =\prod_{j=1}^{\infty} \frac{\left(1+(x q)^{\left\lceil\frac{j}{2}\right\rceil}(x q)^{\left\lfloor\frac{j}{2}\right\rfloor}\left(x^{-1} q\right)^{\left\lceil\frac{j-1}{2}\right\rceil}\left(x^{-1} q\right)^{\left\lfloor\frac{j-1}{2}\right\rfloor}\right)}{\left(1-(x q)^{\left\lceil\frac{j}{2}\right\rceil}(x q)^{\left\lfloor\frac{j}{2}\right\rfloor}\left(x^{-1} q\right)^{\left\lceil\frac{j}{2}\right\rceil}\left(x^{-1} q\right)^{\left\lfloor\frac{j}{2}\right\rfloor}\right)\left(1-(x q)^{j}(x q)^{j}\left(x^{-1} q\right)^{j-1}\left(x^{-1} q\right)^{j-1}\right)} \\
& \quad \times \prod_{r \in \mathbb{N}}\left(1-(x q)^{\left\lceil\frac{r}{2}\right\rceil \frac{2 m+2}{2}}(x q)^{\left\lfloor\frac{r}{2}\right\rfloor \frac{2 m+2}{2}}\left(x^{-1} q\right)^{\left\lceil\frac{r}{2}\right\rceil \frac{2 m+2}{2}}\left(x^{-1} q\right)^{\left\lfloor\frac{r}{2}\right\rfloor \frac{2 m+2}{2}}\right) \\
& =\frac{\prod_{j=1}^{\infty}\left(1+(x q)^{j}(x q)^{j}\left(x^{-1} q\right)^{j}\left(x^{-1} q\right)^{j-1}\right) \prod_{j=1}^{\infty}\left(1+(x q)^{j}(x q)^{j-1}\left(x^{-1} q\right)^{j-1}\left(x^{-1} q\right)^{j-1}\right)}{\prod_{j=1}^{\infty}\left(1-(x q)^{\left\lceil\frac{j}{2}\right\rceil}(x q)^{\left\lfloor\frac{j}{2}\right\rfloor}\left(x^{-1} q\right)^{\left\lceil\frac{j}{2}\right\rceil}\left(x^{-1} q\right)^{\left\lfloor\frac{j}{2}\right\rfloor}\right) \prod_{j=1}^{\infty}\left(1-x^{2} q^{4 j-2}\right)}
\end{aligned}
$$




$$
\begin{aligned}
& \times \prod_{r \in \mathbb{N}}\left(1-(x q)^{\left\lceil\frac{r}{2}\right\rceil(m+1)}(x q)^{\left\lfloor\frac{r}{2}\right\rfloor(m+1)}\left(x^{-1} q\right)^{\left\lceil\frac{r}{2}\right\rceil(m+1)}\left(x^{-1} q\right)^{\left\lfloor\frac{r}{2}\right\rfloor(m+1)}\right) \\
= & \frac{\prod_{j=1}^{\infty}\left(1+x q^{4 j-1}\right) \prod_{j=1}^{\infty}\left(1+x q^{4 j-3}\right)}{\prod_{j=1}^{\infty}\left(1-q^{4 j}\right)\left(1-q^{4 j-2}\right) \prod_{j=1}^{\infty}\left(1-x^{2} q^{4 j-2}\right)} \times \prod_{r=1}^{\infty}\left(1-q^{4 r(m+1)}\right)\left(1-q^{(4 r-2)(m+1)}\right) \\
= & \frac{\left(-x q ; q^{2}\right)_{\infty}\left(q^{2 m+2} ; q^{2 m+2}\right)_{\infty}}{\left(q^{2} ; q^{2}\right)_{\infty}\left(x^{2} q^{2} ; q^{4}\right)_{\infty}} .
\end{aligned}
$$

Meanwhile, setting $a=x q, b=x^{-1} q, R=E$ (the set of even positive integers) and $\gamma=m$ in Theorem 2.4, we find that

$$
\begin{aligned}
& \Psi_{E, m}\left(x q, x^{-1} q, x q, x^{-1} q\right) \\
= & \prod_{j=1}^{\infty} \frac{\left(1+(x q)^{\left\lceil\frac{j}{2}\right\rceil}\left(x^{-1} q\right)^{\left\lfloor\frac{j}{2}\right\rfloor}(x q)^{\left\lceil\frac{j-1}{2}\right\rceil}\left(x^{-1} q\right)^{\left\lfloor\frac{j-1}{2}\right\rfloor}\right)}{\left(1-(x q)^{2\left\lceil\frac{j}{2}\right\rceil}\left(x^{-1} q\right)^{2\left\lfloor\frac{j}{2}\right\rfloor}\right)\left(1-(x q)^{2 j-1}\left(x^{-1} q\right)^{2 j-1}\right)} \\
& \times \prod_{r \in E}\left(1-(x q)^{\left\lceil\frac{r}{2}\right\rceil(m+1)}\left(x^{-1} q\right)^{\left\lfloor\frac{r}{2}\right\rfloor(m+1)}\right) \\
= & \frac{\prod_{j=1}^{\infty}\left(1+(x q)^{j}\left(x^{-1} q\right)^{j}(x q)^{j}\left(x^{-1} q\right)^{j-1}\right) \prod_{j=1}^{\infty}\left(1+(x q)^{j}\left(x^{-1} q\right)^{j-1}(x q)^{j-1}\left(x^{-1} q\right)^{j-1}\right)}{\prod_{j=1}^{\infty}\left(1-(x q)^{2\left\lceil\frac{j}{2}\right\rceil}\left(x^{-1} q\right)^{2\left\lfloor\frac{j}{2}\right\rfloor}\right) \prod_{j=1}^{\infty}\left(1-q^{4 j-2}\right)} \\
= & \frac{\prod_{j=1}^{\infty}\left(1+x q^{4 j-1}\right) \prod_{j=1}^{\infty}\left(1+x q^{4 j-3}\right)}{\prod_{j=1}^{\infty}\left(1-q^{4 j}\right)\left(1-x^{2} q^{4 j-2}\right) \prod_{j=1}^{\infty}\left(1-q^{4 j-2}\right)} \times \prod_{r=1}^{\infty}\left(1-q^{2 r(m+1)}\right) \\
= & \frac{\left(-x q ; q^{2}\right)_{\infty}\left(q^{2 m+2} ; q^{2 m+2}\right)_{\infty}}{\left(q^{2} ; q^{2}\right)_{\infty}\left(x^{2} q^{2} ; q^{4}\right)_{\infty}} .
\end{aligned}
$$

This completes the proof.

Similarly, one can check that

$$
\Phi_{E, 2 m+2}\left(x q, x q, x^{-1} q, x^{-1} q\right)
$$

is the generating function for partitions such that each even part appears at most $2 m+1$ times and the exponent of $x$ is the alternating sum. Moreover, one sees that

$$
\Psi_{E, 2 m+1}\left(x q, x^{-1} q, x q, x^{-1} q\right)
$$

is the generating function for partitions such that each even part appears at most $2 m+$ 1 times and the exponent of $x$ is the number of odd parts. Thus Theorem 3.2 is a consequence of the following relation. 
Theorem 3.4. We have

$\Phi_{E, 2 m+2}\left(x q, x q, x^{-1} q, x^{-1} q\right)=\Psi_{E, 2 m+1}\left(x q, x^{-1} q, x q, x^{-1} q\right)=\frac{\left(-x q ; q^{2}\right)_{\infty}\left(q^{4 m+4} ; q^{4 m+4}\right)_{\infty}}{\left(q^{2} ; q^{2}\right)_{\infty}\left(x^{2} q^{2} ; q^{4}\right)_{\infty}}$.

Proof. Making the substitutions $a=x q, b=x q, R=E$ (the set of even positive integers) and $\rho=2 m+2$ in Theorem 2.3, we find that

$$
\begin{aligned}
& \Phi_{E, 2 m+2}\left(x q, x q, x^{-1} q, x^{-1} q\right) \\
& =\prod_{j=1}^{\infty} \frac{\left(1+(x q)^{\left\lceil\frac{j}{2}\right\rceil}(x q)^{\left\lfloor\frac{j}{2}\right\rfloor}\left(x^{-1} q\right)^{\left\lceil\frac{j-1}{2}\right\rceil}\left(x^{-1} q\right)^{\left\lfloor\frac{j-1}{2}\right\rfloor}\right)}{\left(1-(x q)^{\left\lceil\frac{j}{2}\right\rceil}(x q)^{\left\lfloor\frac{j}{2}\right\rfloor}\left(x^{-1} q\right)^{\left\lceil\frac{j}{2}\right\rceil}\left(x^{-1} q\right)^{\left\lfloor\frac{j}{2}\right\rfloor}\right)\left(1-(x q)^{j}(x q)^{j}\left(x^{-1} q\right)^{j-1}\left(x^{-1} q\right)^{j-1}\right)} \\
& \times \prod_{r \in E}\left(1-(x q)^{\left\lceil\frac{r}{2}\right\rceil \frac{2 m+2}{2}}(x q)^{\left\lfloor\frac{r}{2}\right\rfloor \frac{2 m+2}{2}}\left(x^{-1} q\right)^{\left\lceil\frac{r}{2}\right\rceil \frac{2 m+2}{2}}\left(x^{-1} q\right)^{\left\lfloor\frac{r}{2}\right\rfloor \frac{2 m+2}{2}}\right) \\
& =\frac{\prod_{j=1}^{\infty}\left(1+(x q)^{j}(x q)^{j}\left(x^{-1} q\right)^{j}\left(x^{-1} q\right)^{j-1}\right) \prod_{j=1}^{\infty}\left(1+(x q)^{j}(x q)^{j-1}\left(x^{-1} q\right)^{j-1}\left(x^{-1} q\right)^{j-1}\right)}{\prod_{j=1}^{\infty}\left(1-(x q)^{\left\lceil\frac{j}{2}\right\rceil}(x q)^{\left\lfloor\frac{j}{2}\right\rfloor}\left(x^{-1} q\right)^{\left\lceil\frac{j}{2}\right\rceil}\left(x^{-1} q\right)^{\left\lfloor\frac{j}{2}\right\rfloor}\right) \prod_{j=1}^{\infty}\left(1-x^{2} q^{4 j-2}\right)} \\
& \times \prod_{r=1}^{\infty}\left(1-(x q)^{r(m+1)}(x q)^{r(m+1)}\left(x^{-1} q\right)^{r(m+1)}\left(x^{-1} q\right)^{r(m+1)}\right) \\
& =\frac{\prod_{j=1}^{\infty}\left(1+x q^{4 j-1}\right) \prod_{j=1}^{\infty}\left(1+x q^{4 j-3}\right)}{\prod_{j=1}^{\infty}\left(1-q^{4 j}\right)\left(1-q^{4 j-2}\right) \prod_{j=1}^{\infty}\left(1-x^{2} q^{4 j-2}\right)} \times \prod_{r=1}^{\infty}\left(1-q^{4 r(m+1)}\right) \\
& =\frac{\left(-x q ; q^{2}\right)_{\infty}\left(q^{4 m+4} ; q^{4 m+4}\right)_{\infty}}{\left(q^{2} ; q^{2}\right)_{\infty}\left(x^{2} q^{2} ; q^{4}\right)_{\infty}} \text {. }
\end{aligned}
$$

On the other hand, making the substitutions $a=x q, b=x^{-1} q, R=E$ and $\gamma=2 m+1$ in Theorem 2.4, we get

$$
\begin{aligned}
& \Psi_{E, 2 m+1}\left(x q, x^{-1} q, x q, x^{-1} q\right) \\
& =\prod_{j=1}^{\infty} \frac{\left(1+(x q)^{\left\lceil\frac{j}{2}\right\rceil}\left(x^{-1} q\right)^{\left\lfloor\frac{j}{2}\right\rfloor}(x q)^{\left\lceil\frac{j-1}{2}\right\rceil}\left(x^{-1} q\right)^{\left\lfloor\frac{j-1}{2}\right\rfloor}\right)}{\left(1-(x q)^{2\left\lceil\frac{j}{2}\right\rceil}\left(x^{-1} q\right)^{2\left\lfloor\frac{j}{2}\right\rfloor}\right)\left(1-(x q)^{2 j-1}\left(x^{-1} q\right)^{2 j-1}\right)} \\
& \quad \times \prod_{r \in E}\left(1-(x q)^{\left\lceil\frac{r}{2}\right\rceil(2 m+1+1)}\left(x^{-1} q\right)^{\left\lfloor\frac{r}{2}\right\rfloor(2 m+1+1)}\right) \\
& =\frac{\prod_{j=1}^{\infty}\left(1+(x q)^{j}\left(x^{-1} q\right)^{j}(x q)^{j}\left(x^{-1} q\right)^{j-1}\right) \prod_{j=1}^{\infty}\left(1+(x q)^{j}\left(x^{-1} q\right)^{j-1}(x q)^{j-1}\left(x^{-1} q\right)^{j-1}\right)}{\prod_{j=1}^{\infty}\left(1-(x q)^{2\left[\frac{j}{2}\right\rceil}\left(x^{-1} q\right)^{2\left\lfloor\frac{j}{2}\right\rfloor}\right) \prod_{j=1}^{\infty}\left(1-q^{4 j-2}\right)} \\
& \quad \times \prod_{r=1}^{\infty}\left(1-(x q)^{2 r(m+1)}\left(x^{-1} q\right)^{2 r(m+1)}\right) \\
& =\frac{\prod_{j=1}^{\infty}\left(1+x q^{4 j-1}\right) \prod_{j=1}^{\infty}\left(1+x q^{4 j-3}\right)}{\prod_{j=1}^{\infty}\left(1-q^{4 j}\right)\left(1-x^{2} q^{4 j-2}\right) \prod_{j=1}^{\infty}\left(1-q^{4 j-2}\right)} \times \prod_{r=1}^{\infty}\left(1-q^{4 r(m+1)}\right)
\end{aligned}
$$




$$
=\frac{\left(-x q ; q^{2}\right)_{\infty}\left(q^{4 m+4} ; q^{4 m+4}\right)_{\infty}}{\left(q^{2} ; q^{2}\right)_{\infty}\left(x^{2} q^{2} ; q^{4}\right)_{\infty}} .
$$

This completes the proof.

\section{Combinatorial Proof of Theorem 3.1}

In this section, we present a combinatorial proof of Theorem 3.1. We need a property of Sylvester's bijection between the set $\mathcal{D}(n)=\{\lambda:|\lambda|=n$ and the parts of $\lambda$ are distinct $\}$ and the set $\mathcal{O}(n)=\{\lambda:|\lambda|=n$ and all parts of $\lambda$ are odd $\}$, see also Pak [15]. Bessenrodt [6] showed that Sylvester's bijection maps a partition $\lambda \in \mathcal{D}(n)$ to a partition $\tau \in \mathcal{O}(n)$ such that

$$
\lambda_{1}=l(\tau)+\left(\tau_{1}-1\right) / 2
$$

and the alternating sum of $\lambda$ equals the number of odd parts of $\tau$, that is,

$$
l_{a}(\lambda)=l_{o}(\tau)
$$

We shall give a bijection $\Psi$ between the set $A_{m}(n)$ of partitions of $n$ such that each part appears at most $2 m+1$ times and the set $B_{m}(n)$ of partitions of $n$ such that each even part appears at most $m$ times. Moreover, for any partition $\alpha$ in $A_{m}(n)$, we show that the alternating sum of $\alpha$ is equal to the number of odd parts of $\Psi(\alpha)$ in $B_{m}(n)$.

Let $\alpha$ be a partition in $A_{m}(n)$. The map $\Psi$ from $A_{m}(n)$ to $B_{m}(n)$ can be described as follows.

1. Write $\alpha$ as a bipartition $\phi_{1}(\alpha)=(\lambda, \mu)$ subject to the following conditions. First, set $\lambda$ and $\mu$ to be the empty partition. For each part $t$ of $\alpha$, if $t$ appears an odd number times, then add one part $t$ to $\lambda$ and move the remaining parts $t$ to $\mu$; otherwise, move all parts $t$ of $\alpha$ to $\mu$. Clearly, $\lambda$ is a partition with distinct parts and each part in $\mu$ appears an even number times. Moreover, each part of $\mu$ appears at most $2 m$ times.

2. We apply Sylvester's bijection $\phi_{2}$ to $\lambda$ to obtain a partition $\phi_{2}(\lambda)=\tau$ containing only odd parts.

3. Since each part $t$ in $\mu$ appears an even number times, we merge two parts $t$ into a single part $2 t$. This leads to a partition $\phi_{3}(\mu)=\nu$ consisting of even parts such that each even part appears at most $m$ times.

4. Putting the parts of $\tau$ and $\nu$ together, we obtain a partition $\phi_{4}(\tau, \nu)=\beta$. It is clear that $\beta \in B_{m}(n)$.

Let $\beta$ be a partition in $B_{m}(n)$. The inverse map $\Psi^{-1}$ from $B_{m}(n)$ to $A_{m}(n)$ can be described as follows. 
1. Write $\beta$ as a bipartition $\phi_{4}^{-1}(\beta)=(\tau, \nu)$, where $\tau$ consists of the odd parts of $\beta$ and $\nu$ consists of the even parts of $\beta$. Since each even part of $\beta$ appears at most $m$ times, the multiplicity of each part in $\nu$ does not exceed $m$.

2. Decompose each even part $2 t$ of $\nu$ into two equal parts $t$. We obtain a partition $\phi_{3}^{-1}(\nu)=\mu$ such that each part in $\mu$ appears an even number times. Clearly, each part of $\mu$ appears at most $2 m$ times.

3. Since $\tau$ is a partition with odd parts, we can apply Sylvester's bijection $\phi_{2}^{-1}$ to $\tau$ to obtain a partition $\phi_{2}^{-1}(\tau)=\lambda$ with distinct parts.

4. Putting the parts of $\lambda$ and $\mu$ together, we obtain a partition $\phi_{1}^{-1}(\lambda, \mu)=\alpha$. It is easy to see that $\alpha \in A_{m}(n)$.

It is readily seen that the above map $\Psi$ is a bijection. Here is an example for $m=2$ and $n=48$. Let

$$
\alpha=(1,2,2,2,2,2,4,4,4,4,7,7,7) \in A_{2}(48) .
$$

We decompose $\alpha$ into a bipartition

$$
\phi_{1}(\alpha)=(\lambda, \mu)=((1,2,7),(2,2,2,2,4,4,4,4,7,7)) .
$$

Then we apply Sylvester's bijection to $\lambda$ to obtain

$$
\phi_{2}(\lambda)=\tau=(1,1,1,1,3,3)
$$

Merging equal parts in $\mu$, we get

$$
\phi_{3}(\mu)=\nu=(4,4,8,8,14) .
$$

Finally, putting the parts of $\tau$ and $\mu$ together we obtain

$$
\phi_{4}(\tau, \nu)=\beta=(1,1,1,1,3,3,4,4,8,8,14) \in B_{2}(48) .
$$

The above bijection $\Psi$ leads to a combinatorial proof of Theorem 3.1.

Proof of Theorem 3.1. For a partition $\alpha \in A_{m}(n)$, let $\beta=\Psi(\alpha) \in B_{m}(n)$. We aim to show that the alternating sum of $\alpha$ is equal to the number of odd parts in $\beta$.

For $\phi_{1}(\alpha)=(\lambda, \mu)$ and any part in $\mu$ appears an even number times in $\mu$, the alternating sum of $\alpha$ is equal to the alternating sum of $\lambda$. Using Bessenrodt's refinement [6], it is easy to see that the alternating sum of $\lambda$ is equal to the number of odd parts in $\tau$. Since all the parts in $\nu$ are even numbers, the number of odd parts in $\tau$ is just the number of odd parts of $\beta$. Consequently, we see that the alternating sum of $\alpha$ is equal to the number of odd parts in $\beta$. Hence Theorem 3.1 holds. 
We conclude with a stronger version of Theorem 3.1 based on the above bijection $\Psi$. Let $\mathcal{A}_{\varphi}(n)$ be the set of partitions of $n$ such that the number of appearances of part $i$ is at most $2 \varphi(i)+1$, for any $i>0$. Let $\mathcal{B}_{\varphi}(n)$ be the set of partitions of $n$ such that the number of appearances of part $2 i$ is at most $\varphi(i)$, for any $i>0$. Notice that for any map $\varphi$ from $\mathbb{N}$ to $\mathbb{N}$, the bijection $\Psi$ can be applied to $\mathcal{A}_{\varphi}(n)$ and $\mathcal{B}_{\varphi}(n)$.

Let $p(\lambda)$ denote the largest odd part in $\lambda$, and let $q(\lambda)$ denote the largest part of $\lambda$ with odd multiplicity. Then we have the following correspondence.

Theorem 4.1. Let $P(n, \varphi, i, k, t)$ be the set of partitions $\alpha \in \mathcal{A}_{\varphi}(n)$ of $n$ such that the number of appearances of $i$ in $\alpha$ is at most $2 \varphi(i)+1, l_{a}(\alpha)=k$, and $q(\alpha)=t$. Let $Q(n, \varphi, i, k, t)$ be the set of partitions $\beta \in \mathcal{B}_{\varphi}(n)$ of $n$ such that the number of appearances of $2 i$ in $\beta$ is at most $\varphi(i), l_{o}(\beta)=k$, and $l_{o}(\beta)+(p(\beta)-1) / 2=t$. Then for $i \geqslant 1$ and $k, t \geqslant 0$, there exists a bijection between $P(n, \varphi, i, k, t)$ and $Q(n, \varphi, i, k, t)$.

Proof. Let $\alpha \in P(n, \varphi, i, k, t)$, that is, the number of appearances of $i$ in $\alpha$ is at most $2 \varphi(i)+1, l_{a}(\alpha)=k$, and $q(\alpha)=t$. Let $\Psi$ be the map from $\mathcal{A}_{\varphi}(n)$ to $\mathcal{B}_{\varphi}(n)$. Denote $\Psi(\alpha)$ by $\beta$. We wish to show that $\beta \in Q(n, \varphi, i, k, t)$, that is, the number of appearances of $2 i$ in $\beta$ is at most $\varphi(i), l_{o}(\beta)=k$, and $l_{o}(\beta)+(p(\beta)-1) / 2=t$.

First, write $\alpha$ as a bipartition $\phi_{1}(\alpha)=(\lambda, \mu)$ based on the following procedure. Set the initial values of $\lambda$ and $\mu$ to be the empty partition. For each part $t$ of $\alpha$, if $t$ appears an odd number of times in $\alpha$, then add a part $t$ to $\lambda$ and move the remaining parts $t$ of $\alpha$ to $\mu$; otherwise, move all parts $t$ of $\alpha$ to $\mu$. Now, $\lambda$ is a partition with distinct parts with $\lambda_{1}=q(\alpha)=t$. Moreover, since any part $i$ of $\alpha$ appears at most $2 \varphi(i)+1$ times, the part $i$ in $\mu$ appears at most $2 \varphi(i)$ times. It is evident that $l_{a}(\lambda)=l_{a}(\alpha)=k$.

Since $\lambda$ is a partition with distinct parts, we can apply Sylvester's bijection $\phi_{2}$ to $\lambda$ to obtain a partition of odd parts. Let $\tau=\phi_{2}(\lambda)$. Recall that $l(\tau)+\left(\tau_{1}-1\right) / 2=\lambda_{1}=t$ and $l(\tau)=l_{a}(\lambda)=k$, as given in (4.8) and (4.9). Furthermore, we can merge every two equal parts in $\mu$ to form a partition with even parts, denoted $\nu=\phi_{3}(\mu)$. Observe that the number of appearances of part $2 i$ in $\nu$ is at most $\varphi(i)$.

Finally, we put the parts of $\tau$ and $\nu$ together to form a partition $\beta=\phi_{4}(\tau, \nu)$. It can be seen that the number of appearances of part $2 i$ in $\beta$ is at most $\varphi(i)$ and $l_{o}(\beta)=l(\tau)=k$. Since $\nu$ is partition with even parts, it is easy to see that $p(\beta)=\tau_{1}$. Using the facts $l_{o}(\beta)=l(\tau), p(\beta)=\tau_{1}$ and $l(\tau)+\left(\tau_{1}-1\right) / 2=t$, we obtain that $l_{o}(\beta)+(p(\beta)-1) / 2=t$. This completes the proof.

\section{Combinatorial Proof of Theorem 3.2}

In this section, we prove Theorem 3.2 combinatorially. We perform the same separation procedure $\phi_{1}$ described in Step 1 of the bijection $\Psi$ on a partition $\alpha \in B_{2 m+1}(n)$ to obtain a pair $(\lambda, \mu)$, where $\lambda$ is a partition into distinct parts and $\mu$ is a partition into parts with even multiplicities. We then apply Sylvester's bijection $\phi_{2}$ described in Step 2 of $\Psi$ to $\lambda$ and denote the resulting partition by $\tau$. For $\mu$, we take each of the odd parts $2 i-1$ and 
write its multiplicity $m_{2 i-1}$, which is even, as the sum of powers of 2 , namely,

$$
m_{2 i-1}=a_{1} 2^{1}+a_{2} 2^{2}+\cdots
$$

where $a_{j}=0$ or 1 . We map the copies of odd parts $2 i-1$ from $\mu$ to $2^{j}(2 i-1)$ with multiplicity $a_{j}$ for $j \geqslant 1$. Since $a_{j}=0$ or 1 , at most one copy of each even part can be obtained. Combining these with the even parts of $\mu$, we obtain a partition $\nu$ into even parts with multiplicity at most $2 m+1$. We define $\beta$ to be the partition whose parts are the union of the parts of $\tau$ and $\nu$.

We now show that the map defined above is indeed a bijection. Since the only difference from the proof of Theorem 3.1 is the map for $\mu$, it is sufficient to show that this map is reversible. Note that every even integer can be uniquely written as $2^{j}(2 i-1)$ for some positive integers $i$ and $j$. Thus, given a partition $\nu$ into even parts $2^{j}(2 i-1)$ with multiplicities $m_{2^{j}(2 i-1)}$ not exceeding $2 m+1$, if $m_{2^{j}(2 i-1)}$ is odd, then we transform one copy of the part $2^{j}(2 i-1)$ into $2^{j}$ copies of the part $2 i-1$. If $m_{2^{j}(2 i-1)}$ is even, then it is less than $2 m+1$ and we do nothing. Thus, we obtain a partition $\mu$, where each

part occurs an even number times and each even parts occurs at most $2 m$ times. This completes the proof.

\section{Acknowledgements}

This work was supported by the 973 Project, the PCSIRT Project of the Ministry of Education, and the National Science Foundation of China.

\section{References}

[1] H.L. Alder, Partition identities - From Euler to the present, Amer. Math. Monthly 76 (1969), 733-746.

[2] G.E. Andrews, On generalizations of Euler's partition theorem, Michigan Math. J. 1 (1966), 491-498.

[3] G.E. Andrews, Partition identities, Adv. Math. 9 (1972), 10-51.

[4] G.E. Andrews, The Theory of Partitions, Addison-Wesley Publishing Co., 1976.

[5] G.E. Andrews and K. Eriksson, Integer Partitions, Cambridge Unversity Press, 2004.

[6] C. Bessenrodt, A bijection for Lebesgue's partition identity in the spirit of Sylvester, Discrete Math. 132 (1994), 1-10.

[7] M. Bousquet-Mélou and K. Eriksson, Lecture hall partitions, Ramanujan J. 1 (1997), $101-111$.

[8] M. Bousquet-Mélou and K. Eriksson, Lecture hall partitions II, Ramanujan J. 1 (1997), 165-185.

[9] C. Boulet, A four parameter partition identity, Ramanujan J. 12(3) (2006), 315-320. 
[10] W.Y.C. Chen, H.Y. Gao, K.Q. Ji and M.Y.X. Li, A unification of two refinements of Euler's partition theorem, Ramanujan J. 23 (1) (2010), 137-149.

[11] N.J. Fine, Basic Hypergeometric Series and Applications, Math. Surveys 27, AMS Providence, 1988.

[12] J.W.L. Glaisher, A theorem in partitions, Messenger of Math. 12 (1883), 158-170.

[13] G. Gasper and M. Rahman, Basic Hypergeometric Series, Encycl. of Math. and Its Appl., Vol. 35, Cambridge University Press, Cambridge, 1990.

[14] E. Moore, Generalized Euler-type partition identities, J. Combin. Theory Ser. A 17 (1974), 78-83.

[15] I. Pak, Partition bijections, a survey, Ramanujan J. 12(1) (2006), 5-75.

[16] J. Sylvester, A constructive theory of partitions, arranged in three acts, an interact and an exodion, Amer. J. Math. 5 (1882), 251-330. 